\title{
L'atelier d'écriture comme dispositif de diagnostic et de développement de la compétence scripturale : du centre de formations d'adultes à la classe
}

Writing Workshop as a Device to Identify and Promote Writing Skills: From an Adult Educational Center to School

\section{Maurice Niwese et Sandrine Bazile}

\section{OpenEdition}

Journals

\section{Édition électronique}

URL : https://journals.openedition.org/pratiques/2063

DOI : 10.4000/pratiques.2063

ISSN : 2425-2042

\section{Éditeur}

Centre de recherche sur les médiations (CREM)

Édition imprimée

Date de publication : 5 juin 2014

Référence électronique

Maurice Niwese et Sandrine Bazile, «L'atelier d'écriture comme dispositif de diagnostic et de développement de la compétence scripturale : du centre de formations d'adultes à la classe » Pratiques [En ligne], 161-162 | 2014, mis en ligne le 05 juin 2014, consulté le 21 septembre 2021. URL : http://journals.openedition.org/pratiques/2063 ; DOI : https://doi.org/10.4000/pratiques.2063

Ce document a été généré automatiquement le 21 septembre 2021.

(c) Tous droits réservés 


\section{L'atelier d'écriture comme dispositif de diagnostic et de développement de la compétence scripturale : du centre de formations d'adultes à la classe}

Writing Workshop as a Device to Identify and Promote Writing Skills: From an Adult Educational Center to School

Maurice Niwese et Sandrine Bazile

1 À la suite notamment des changements sociologiques qui ont affecté l'école, celle-ci accueille de plus en plus des populations hétérogènes (Verhoeven, 2002), dont la maitrise de la langue écrite est défaillante, alors que celle-ci participe à la construction des savoirs dans toutes les disciplines et qu'elle constitue un facteur de réussite scolaire et sociale (Reuter, 2002 ; Niwese, 2010). Cette hétérogénéité du public ne permet pas de déterminer a priori les compétences de celui-ci, alors que l'efficacité des interventions didactiques reste tributaire de la connaissance des besoins des apprenants.

2 La recherche présentée dans cette contribution fait partie d'un projet global qui, à terme, vise à construire une méthodologie de recherche permettant d'identifier les difficultés et les compétences en matière d'écriture dans des classes hétérogènes, auprès des élèves de fin du cycle 3 et de début du cycle d'adaptation, au collège. Le choix de ce niveau de scolarité s'est avéré pertinent pour deux raisons au moins. Ce niveau, qui est marqué par une progression sensible des activités d'écriture tant du point de vue de leurs modalités que de leur spécialisation générique, apparait comme charnière au regard de la validation du palier 2 du socle commun de connaissances et de compétences (première année du palier $3 \mathrm{du}$ socle) et semble révéler des situations d'hétérogénéité. En outre, en proposant de regrouper d'ici 2017 le CM1, le CM2 et la sixième dans un même cycle (le cycle 3 dit de « consolidation »), le récent décret relatif 
à la réorganisation des cycles d'enseignement en primaire et au collège ${ }^{1}$ oblige à repenser la continuité de la progression des apprentissages.

Cet article portera principalement sur un dispositif d'atelier d'écriture favorisant la réécriture et socialisant les textes produits, qui a été expérimenté en Belgique auprès d'un public adulte faiblement scolarisé et socialement défavorisé (Niwese, 2010), dispositif en cours de transposition en milieu scolaire français auprès du public indiqué ci-dessus. Après la présentation du dispositif, nous montrerons d'abord comment celuici permet de travailler la compétence scripturale (Lafont-Terranova, 2009) dans toutes ses composantes (Barré-De Miniac, 2000) et verrons ensuite comment une analyse génétique de textes (Grésillon, 1994), à l'aide notamment du logiciel MEDITE (Ganascia, Fenoglio \& Lebrave, 2004), peut contribuer à déterminer les difficultés et les compétences des scripteurs en matière d'écriture. Nous décrirons enfin la première phase du processus de transposition du dispositif à l'école avant de rendre compte de quelques résultats de cette étape.

\section{Présentation du dispositif}

4 Comme indiqué ci-dessus, le dispositif présenté ici a été expérimenté auprès d'un public hétérogène (par ses origines culturelles, son parcours scolaire et biographique, ses projets de vie, ses pratiques linguistiques et scripturales, etc.), en difficulté scripturale (maitrise défaillante de l'écriture, représentations de l'écriture en décalage avec la réalité, etc.) et identitaire (déficit d'estime de soi, méfiance vis-à-vis d'autrui, sentiments d'exclusion sociale, etc.). Ce public était inscrit dans des centres bruxellois de formation d'adultes en vue d'une insertion socioprofessionnelle. Nous avons suivi 30 cas, dont 10 dans la phase exploratoire et 20 au cours de l'expérimentation. Le profil du public nous ${ }^{2}$ a conduit à travailler sur le développement aussi bien personnel (rapport à soi et à autrui) que scriptural (rapport à l'écriture, identification du déjà-là, etc.) via un atelier d'écriture centré sur la production d'un texte narratif de type autobiographique (dit aussi récit de soi) et accordant une attention particulière à la réécriture et à la socialisation des textes produits. L'atelier mis en place rompt avec ce qui se pratique généralement. En effet, «la plupart des ateliers portant sur le développement de la compétence scripturale sont adressés à des populations favorisées ", alors que, pour celles qui sont " en difficulté, on a tendance à ne viser que la valorisation de soi » (Niwese, 2012 : 124).

\subsection{Choix d'un atelier d'écriture}

5 Le choix d'un atelier d'écriture a été motivé par le fait que des travaux menés en didactique de l'écriture montrent que ce dispositif a des effets positifs sur le texte et sur son auteur (Lafont-Terranova, 2009). Dans ce sens, il aide ceux qui en bénéficient à prendre confiance en eux-mêmes, à oser écrire, voire à mieux écrire.

6 L'expérimentation du dispositif a été réalisée en trois phases: la mise en route, la production du récit et l'évaluation.

7 La première phase a consisté à créer les conditions favorables à la production du récit en développant la cohésion entre les participants, en identifiant et en travaillant leurs représentations sur l'écriture, en renforçant la confiance en leurs capacités d'écriture et en les initiant à la production de différents types de textes. Au cours de cette phase, 
nous avons, par exemple, proposé des pistes d'écriture centrées sur le vécu, fait produire des textes courts, négocié un contrat de groupe (intégrant la manière de réagir aux textes des pairs) et travaillé à partir de brouillons et de témoignages des scripteurs experts.

La deuxième phase a été consacrée à la production du récit. Alors que la première phase jouait le rôle d'initiation tant du point de vue thématique que narratif, la deuxième a réinvesti ce qui avait été commencé en l'approfondissant et en le complexifiant. Contrairement à la première phase, la deuxième a connu un temps de réécriture, en plus des temps de motivation, de production, de lecture et de réception des textes produits.

9 Par le truchement d'un questionnaire, des entretiens individuels et de la production d'une postface portant sur l'expérience vécue dans l'atelier, la troisième phase a cherché à déterminer avec les participants ce qui avait été acquis et ce qui restait à faire.

\subsection{Un atelier d'écriture favorisant la réécriture}

10 Ce sont les bénéfices didactiques qui nous ont poussés à opter pour un dispositif qui favorise la réécriture entendue à la fois comme réécriture génétique, c'est-à-dire tout retour sur son propre texte ou sur celui d'un tiers pour l'améliorer, l'adapter à d'autres textes ou à d'autres publics, et comme récriture intertextuelle, à savoir toute transposition d'un texte initial détourné ou parodié (Gignoux, 2006; Connan-Pintado, 2010).

11 La réécriture offre en effet au formateur la possibilité de déterminer ce que le scripteur sait faire seul ou avec l'aide d'un tiers et au scripteur de s'autocorriger en évitant des erreurs dues à une gestion simultanée de toutes les tâches que requiert l'acte d'écriture (Reuter, 2002). Elle permet aussi de lutter contre une représentation fixiste du texte en mettant l'accent sur «le caractère dynamique intrinsèque à l'écriture " (Fenoglio \& Boucheron-Pétillon, 2002: 5), ce qui valorise le processus de production prenant ainsi le contrepied d'une vision archaïque selon laquelle « raturer, c'est rater son travail [et] réussir, c'est pouvoir rendre sa copie au premier jet, c'est travailler vite et bien » (Bucheton, 1992 : 130).

12 Si réécrire représente un intérêt indéniable pour la didactique de l'écriture, (faire) réécrire ne va pas de soi. Sans travail préalable et sans raisons valables, «la réécriture peut être vécue comme une contrainte supplémentaire inutile» (Niwese, 2010:61) et comme une « lubie de l'enseignant» (Reuter, $2002: 173)$.

13 La difficulté de réécrire nous a conduit à introduire cette pratique de façon progressive. $\mathrm{Au}$ cours de la première phase, nous nous sommes appuyés sur des productions (intermédiaires et définitives) et sur des témoignages d'écrivains pour faire évoluer les représentations des participants. En considérant les brouillons de grands auteurs, ces derniers se sont rendu compte que « loin de s'inscrire dans une continuité homogène et linéaire ", leur écriture "zigzague» (Fenoglio \& Boucheron-Pétillon, 2002: 5). De même, leurs témoignages montrent qu'eux aussi traversent des moments non seulement de plaisir, mais aussi de doutes et d'angoisse. Nous avons enfin comparé les textes intermédiaires et les textes publiés pour montrer que les textes définitifs gomment le travail de production. 
14 Après le travail sur les représentations, les scripteurs ont d'abord réécrit (en petits groupes) les textes des autres (des pairs ou des participants d'autres ateliers). Ce n'est que plus tard, au cours de la production du récit, qu'ils ont commencé à retravailler leurs propres textes en vue de leur publication en dehors du groupe. Les textes des participants ont connu au moins quatre versions. La première (V1) correspond au premier jet produit et lu au groupe ; après cette version, il a été demandé à chacun d'en préparer une deuxième (V2) à soumettre à la relecture des pairs et du formateur ; avec l'aide $\mathrm{du}$ formateur, le scripteur a préparé une troisième version (V3) qu'il a retranscrite dans un cahier destiné à contenir les textes prêts à être publiés. Corrigés par le formateur, ces derniers textes ont donné lieu à la version publiée (V4).

Il importe de noter que le récit a été produit en quatre temps, à partir des pistes d'écriture que le formateur dévoilait progressivement, ce qui implique que la production d'une nouvelle partie pouvait entrainer la réécriture des parties antérieures pour créer une cohérence d'ensemble. " Produire un même texte en plusieurs temps et en rassemblant l'hétérogène constitue également une bonne occasion de lutter contre de fausses idées qui veulent qu'il faille avoir nécessairement tout le texte dans la tête avant de se mettre à écrire » (Niwese, $2010: 269$ ).

\subsection{Textes narratifs de type autobiographique}

Le dispositif expérimenté visait principalement à faire produire « un texte narratif de type autobiographique dans lequel le personnage principal, s'exprimant en "je", devait correspondre au narrateur, mais différer de l'auteur» (Niwese, 2010:269); récit de type autobiographique parce qu'il ne s'agit pas d'une autobiographie pure supposant la triple identité entre le personnage principal, le narrateur et l'auteur (Lejeune, 1996); de type autobiographique aussi, parce que, à la suite de P. Ricœur (1985), nous rejetons «la fictionnalité pure du récit» en admettant que celui-ci, qu'il soit dit fictif ou historique, reste un « rejeton fragile issu de l'union de l'histoire et de la fiction » (ibid. : 442).

17 Pour notre expérimentation, «même si les scripteurs étaient avisés qu'il n'était pas question d'écrire une autobiographie comprise au sens de Lejeune (1996), nous les avons entrainés sur le terrain de l'autobiographie en leur demandant de mettre en scène le narrateur-personnage principal s'exprimant en “je” " (Niwese, 2012 : 132). C'est cette autobiographie oblique, dont le « je » est officiellement un autre, que nous qualifions de récit de soi ou de texte narratif de type autobiographique.

Le choix du récit de soi a été motivé par le fait qu'il constitue « le lieu où des questions non seulement linguistiques, mais aussi identitaires [peuvent] se rencontrer et être travaillées » (Niwese, $2010: 479$ ).

Par rapport à d'autres sources d'écriture ${ }^{3}$ (« les jeux de la langue et des textes », « les savoirs théorico-réflexifs ", "l'imaginaire et la créativité ») et pour un public ayant un faible bagage culturel, le récit de soi est d'un accès facile parce que notre vécu constitue " un matériau (conscient ou inconscient, immédiatement disponible ou non, organisé ou non) très riche » (Reuter, 2002 : 137). Mais, au-delà de l'accessibilité, en tant que récit, le récit de soi donne la possibilité de travailler plusieurs séquences textuelles (Adam, 2008). Sur le plan personnel, le récit de soi permet de «rester maitre de ses affects sans pour autant les nier ou les refouler» (Bucheton, $1995: 284)$, de se (faire) comprendre et de comprendre l'autre (Niwese, 2012). 


\subsection{Large socialisation des productions} dans un recueil et sur le site web du centre de formation. Cette publication constitue en réalité une troisième socialisation des textes produits, la première étant leur lecture au groupe et la seconde correspondant à la lecture d'extraits du recueil à une assemblée réunie à l'occasion de la publication de celui-ci. Ces socialisations de textes marquent « une rupture forte avec les pédagogies conventionnelles » où « l'écrit n'a guère pour destinataire que le professeur, généralement réduit au rôle de correcteur » (André, 1989 : 69).

21 La socialisation des textes donne du sens à l'acte d'écrire, constituant ainsi « un facteur de motivation, de mobilisation et de valorisation » des scripteurs (Niwese, $2010: 160$ ). Dans le cadre de la pédagogie du projet où nous nous situons (Niwese, $2010 ; 2012$ ), la confection d'un recueil de textes visait la réalisation d'un objet social concret, réalisation qui peut être "vécue par les formés comme un véritable défi qui incit[e] chacun à mobiliser toute son énergie et toutes ses compétences » (Huber, $2005: 42$ ).

\section{Analyse et quelques résultats}

Établis à partir d'une analyse de déclarations et de textes produits, les résultats présentés ci-dessous portent sur les effets du dispositif expérimenté sur la compétence scripturale de ceux qui en ont bénéficié ainsi que sur leur rapport à eux-mêmes et à autrui.

Pour faciliter la lecture des résultats, il importe de rappeler que nous concevons la compétence scripturale au sens des didacticiens de l'écriture (Dabène, 1991; LafontTerranova, 2009) qui la définissent comme englobant le "savoir-graphier» dans ses aspects matériels (outils, supports, tracés de lettres, etc.) et graphiques (relation entre graphèmes et phonèmes), le "savoir-faire textuel " dans ses aspects linguistiques (lexique, morphologie, syntaxe phrastique et textuelle, etc.) et pragmatiques (questions de lisibilité, de mondes rapportés, de visées illocutoires, etc.) ainsi que le «rapport à l'écriture " (Barré-De Miniac, 2000; Chartrand \& Blaser, 2008), incluant les représentations, dans ses dimensions affective (investissement de l'écriture), axiologique (valeurs, opinions et attitudes vis-à-vis de l'écriture), conceptuelle (conception de l'écriture et de son apprentissage) et méta-scripturale (verbalisation de sa propre pratique d'écriture). Quant au rapport à soi et à autrui, nous l'avons défini comme pouvant «traduire soit ce que le sujet pense de lui, soit ce qu'il croit que les autres pensent de lui, soit, enfin, ce qu'il pense des autres » (Niwese, $2010: 52$ ).

\subsection{Effets subjectifs}

Pour déterminer si le dispositif avait permis aux participants d'améliorer leur rapport à l'écriture ainsi que leur rapport à eux-mêmes et à autrui, nous avons analysé leurs déclarations écrites et orales, déclarations recueillies par plusieurs canaux: questionnaire, entretiens individuels, évaluations réalisées tout au long de l'expérimentation, postfaces, etc. 


\subsubsection{Amélioration des relations (inter)personnelles}

Du point de vue personnel, l'analyse a démontré que l'atelier d'écriture «a permis au scripteur de se comprendre et de mieux comprendre l'autre " et qu'il a été perçu comme "un espace-temps où le sujet-écrivant s'est senti écouté par le groupe et encouragé par le formateur " (Niwese, 2010 : 375). Par exemple, dans les deux extraits repris ci-dessous, Albertine comme Fiona reviennent sur des liens de rapprochement interculturel, tissés grâce aux échanges favorisés par l'atelier :

«Par rapport aux autres, je vois qu'en écrivant comme ça et en écoutant les gens, je pense qu'on a beaucoup de points communs » (Albertine, entretiens, 2007).

"Grâce à l'atelier, je me suis sentie proche des autres. De loin, on a souvent une appréhension fausse des autres. Mais en les approchant, [en] conversant avec les différentes personnes, [les] différentes cultures, on comprend mieux la façon de vivre des autres » (Fiona, évaluation d'approfondissement, 2007).

climat de confiance indispensable pour tout atelier d'écriture est davantage nécessaire quand on a affaire à un groupe hétérogène dans lequel l'autre peut être posé comme étant a priori différent.

\subsubsection{Amélioration du rapport à l'écriture}

27 Au niveau axiologique, il ressort des déclarations des participants que ceux-ci ont pris conscience que l'écriture est incontournable dans nos sociétés et que la réussite socioprofessionnelle peut être reliée à sa maitrise. S'en passer, pense par exemple Fiona, était possible à une époque aujourd'hui révolue :

« Nos arrière-parents ne partaient pas à l'école, mais ils vivaient quand même. Mais en nos temps, les choses ont changé. On doit apprendre. Si je ne viens pas ici apprendre à écrire le français, je ne saurai pas suivre une formation qualifiante » (Fiona, entretiens, 2007).

« Il y a [des] métiers qui nécessitent l'écriture. Or, les difficultés en écriture ne permettront pas l'accès à ces métiers " (Fiona, évaluation d'approfondissement, 2007).

Le changement des représentations en rapport avec l'importance de l'écriture n'est pas anodin pour des personnes, dont certaines se posaient des questions sur son utilité. En effet, «l'obstacle à la lecture [mais aussi à l'écriture] ne réside pas d'abord dans un manque de techniques mais dans l'absence de raisons de se doter de ces techniques » (Stercq, $2009: 102$ ).

Pour ce qui est de la dimension affective, nombreux sont les participants qui affirment qu'ils ont (re)pris confiance en leurs capacités d'écrire et qu'ils écrivent davantage et plus facilement qu'auparavant. Dans le passage repris ci-dessous, Fiona explique que l'atelier lui a permis de surmonter ses craintes et d'arriver à écrire sans se laisser paralyser par le risque de commettre des erreurs :

«L'écriture, ça m'a aussi beaucoup aidée, parce que je peux écrire avec fautes, mais sans crainte ; avant, je n'osais pas écrire, j'avais tout le temps honte en moi-même que j'écris en français avec plein de fautes et tout, ça me gênait. Mais maintenant, ça va, je peux écrire avec ou sans fautes, ça m'est égal » (Fiona, entretiens, 2007).

De la peur d'écrire, les participants ont osé écrire et ont découvert qu'ils avaient des capacités, dont ils ne soupçonnaient pas l'existence. Le dispositif a par ailleurs suscité chez certains l'envie de continuer à écrire : 
« Pour moi, l'atelier d'écriture m'a permis de tester mes aptitudes à l'écriture, de créer une petite histoire à partir de l'imaginaire. Ça m'a aussi permis de confirmer mon envie d'écrire. J'ai toujours voulu écrire l'histoire de mon père et grâce à l'atelier d'écriture, l'envie devient plus grande. [...] Ce qui m'a marquée, c'est que j'ai pris conscience que j'étais capable, non pas de devenir un écrivain célèbre, mais [d'écrire] à des fins professionnelles » (Samira, postface).

Sur le plan conceptuel, les participants ont compris qu'on peut apprendre à écrire et que la réécriture est inhérente à tout acte d'écriture. Dans les deux extraits suivants, Tharcisse et Lucien insistent sur la nécessité d'un retour sur le déjà-produit, qu'ils retravaillent à plusieurs reprises, et sur le fait que chaque réécriture améliore le texte :

«À chaque fois que j'ai réécrit [...], j'ai vu toutes les fautes que je faisais au niveau lourdeur et c'est encore un peu mieux, je faisais chaque fois un peu mieux, chaque fois que je le réécrivais. [...] Donc, je suis vraiment obligé de réécrire pour vraiment obtenir ce que je veux » (Tharcisse, entretiens, 2007).

«Parce qu'on forme des phrases et après on essaie de mieux les refaire. En plus, la première fois, on réfléchit pas trop et au fur et à mesure ça s'améliore. [...] Quand j'ai transcrit dans le cahier, la troisième fois, j'ai encore modifié. Donc chaque fois, j'ai un peu amélioré » (Lucien, entretiens, 2007).

Enfin, au niveau métascriptural, les participants ont acquis la capacité « de parler de l'écriture en général et de leurs pratiques scripturales en particulier " (Niwese, 2011 : 39). D'après C. Barré-De Miniac (2000), cette métacognition, très présente dans les verbalisations relatives aux dimensions conceptuelle et métascripturale, joue un grand rôle dans le processus d'apprentissage de l'écriture.

\subsection{Effets textuels : du ressenti au texte}

Après l'analyse des effets relevant du déclaratif, nous nous sommes intéressés aux textes produits par les participants pour déterminer d'abord si le dispositif a réellement permis d'écrire davantage, ensuite comment ces derniers procèdent pour réécrire et, enfin, si la réécriture a été synonyme du mieux-écrire. Utilisant les outils et les notions empruntés à la génétique textuelle (Grésillon, 1994) ainsi que les ressources de la linguistique (Adam, 2008) et de la narratologie (Adam, 1992), nous avons analysé 4 versions de 48 textes produits dans l'atelier, textes dont les premières versions totalisent 12508 mots.

\subsection{1. Évolution du volume de l'expression verbale}

Des auteurs tels N. Gueunier (1993) et J. Lafont (1999) utilisent, mais de façon différente, le volume de l'expression verbale (VET) comme critère d'évaluation de la compétence en écriture. Gueunier (1993) fait produire un texte pendant un temps limité et travaille sur le VET en lien avec le temps de production. Dans ce contexte, le VET est considéré comme « un indicateur partiel de compétence, dans la mesure où le temps de passation étant limité, il manifeste la rapidité d'actualisation : textuelle, syntaxique et lexicale » (ibid. : 196). Ce procédé d'analyse a des limites. Non seulement, comme le dit Gueunier (1993), il y a risque de "verbosité creuse », mais aussi, en plus du temps, le VET peut dépendre de plusieurs facteurs, dont le genre du texte à produire, sa finalité, la situation de sa production, la consigne donnée, etc.

Suivant Lafont (1999), nous nous intéressons, quant à nous, à l'évolution du VET lors du passage d'une version à une autre, évolution que nous déterminons en comparant le 
nombre de mots de la version initiale (VI) à celui de la version réécrite (VR). Si l'écart entre la VI et la VR est important, c'est l'indice du fait que la réécriture n'a pas été une simple mise au propre du déjà-écrit, ce qui, du point de vue du rapport à l'écriture, est à la fois un signe d'investissement dans l'écriture (dimension affective) et un indicateur que le scripteur ne considère pas le premier jet comme une réalité figée et intouchable (dimension conceptuelle). Cette dernière représentation est essentielle pour que le scripteur accepte que son texte puisse être modifié, voire critiqué, sans que cela se répercute négativement sur sa propre personne.

Dans le cadre de l'atelier expérimenté, sur 100 mots réécrits, on a enregistré sur l'ensemble des textes analysés, une augmentation de près de 20 mots. Et pour certains participants, le texte ajouté à la VI a été quantitativement plus important que celle-ci. $\mathrm{Si}$, de façon générale, l'évolution du VET montre que les scripteurs modifient leurs textes en les réécrivant, elle ne donne pas d'éléments sur la nature des transformations réalisées. Or, le type d'opérations de réécriture peut constituer une mesure d'appréciation de ce qui se construit.

\subsubsection{Les opérations de réécriture}

Nous venons d'admettre que l'évolution du VET est une preuve matérielle du fait que les scripteurs ne se limitent pas au premier jet. Or, comme il n'existe pas nécessairement une adéquation entre l'évolution du VET et la nature des transformations réalisées dans le texte réécrit (Niwese, 2010), le VET ne suffit pas. Par exemple, un scripteur peut doubler ou réduire de moitié son texte par une seule opération. De même, « un texte dont le volume évolue à peine peut avoir fait l'objet d'un très grand nombre d'opérations de réécriture et donc avoir été modifié de façon sensible lors de la réécriture" (Lafont, 1999: 457). Pour ces raisons, nous nous intéressons à la fréquence et au statut des opérations de réécriture (ORE).

La fréquence, parce que, plus le taux d'ORE est élevé, plus le texte peut avoir été retravaillé à plusieurs endroits. Le statut, parce que toutes les ORE ne demandent pas au scripteur le même investissement. Selon C. Fabre-Cols (2002), le remplacement est l'ORE la plus pratiquée par les scripteurs non experts qui ont tendance à effectuer « des modifications formelles » (ibid.: 86) ; l'ajout, utilisé par les scripteurs expérimentés, est une ORE coûteuse parce qu'elle suppose d'aller au-delà du déjà-produit en adoptant " une attitude créatrice et non plus seulement méliorative » (ibid. : 85); la suppression suppose un renoncement radical à ce qui a été écrit, ce qui « requiert une attitude distanciée vis-à-vis de l'écriture [...], difficile pour tous les scripteurs, et en particulier les débutants " (ibid.: 110); le déplacement est une ORE complexe, sous-représentée dans les écrits des non-experts, puisqu'elle « implique le traitement simultané des axes paradigmatique et syntaxique » (Niwese, $2010: 392$ ).

Pour repérer et classer les ORE réalisées lors du passage d'une version à une autre, nous utilisons le logiciel MEDITE. L'intérêt de ce logiciel réside dans la systématisation de la démarche et dans l'identification constante des éléments touchés par la réécriture. En effet, par son interface, MEDITE permet d'afficher simultanément en vis-à-vis deux versions d'un même texte, de déterminer avec précision les différentes modifications qui ont eu lieu et, grâce à une troisième fenêtre, de les répertorier par catégories (ibid. : 385-387). 


\section{Du premier jet à la première version réécrite} V2 (premier jet réécrit), entièrement produites par le scripteur pour voir ce qui se passe quand ce dernier retravaille son texte. Nous avons ensuite comparé la V2 et la V4 (version jugée acceptable, qui intègre les corrections réalisées par le formateur) pour déterminer ce qui est déjà là et, surtout, ce qui reste à faire pour que le scripteur produise un récit acceptable.

41 L'analyse comparative de la V1 et de la V2 a montré que les scripteurs ont réécrit profondément leurs textes en diversifiant les ORE : sur 100 mots réécrits, on dénombre 10 ORE, dont $51,53 \%$ sont des remplacements (R), 29,39\% des ajouts (A), 15,42\% des suppressions (S) et 3,66 \% des déplacements (D). En comparant ces résultats avec ceux des études antérieures (Fabre-Cols, 2002), on remarque que le taux d'ORE ainsi que l'ordre dominant $(\mathrm{R}>\mathrm{A}>\mathrm{S}>\mathrm{D})$ placent les bénéficiaires de l'atelier expérimenté du côté des scripteurs familiers de l'écriture. Eu égard au fait que le public d'expérience n'écrivait guère avant d'avoir fréquenté l'atelier, on peut penser que celui-ci lui «a permis d'adopter un comportement comparable à celui des scripteurs qui ne sont pas novices » (ibid. : 392).

Pour mieux comprendre, du point de vue qualitatif, ces différentes modifications, nous avons étudié le fonctionnement des deux ORE les plus représentatives : l'ajout et le remplacement.

Pour l'ajout, au niveau des éléments inférieurs à la proposition-énoncé (Adam, 2008), nous avons trouvé que des ajouts visaient à rétablir des segments oubliés (« $A$ chaque /match ${ }^{4}$, on ma toujours sélectionné " [Albertine]), à apporter des informations nouvelles par l'insertion de mots de type référentiel («Nous habitions, /maman, mes 2 frères, ma sceur et moi/ une belle maison /un peu en dehors de la ville/» [Céline]) ou à repositionner le scripteur dans la prise en charge de son discours («Mais, /Hélas!/ quand on est petit on peut pas décider " [Albertine]). Les ajouts supérieurs à la propositionénoncé consistaient, quant à eux, à contextualiser, expliquer, évaluer ou tout simplement continuer ce qui avait été déjà produit. Par exemple, en réécrivant la fin de son histoire, Céline ajoute (au début du dénouement) ce qui suit: "/Quelque chose d'extraordinaire, m'est arrivé un soir, cela peut paraitre fou mais c'est vraiment réel. Je vais vous partager ce passage de ma vie, croyez y ou pas, je vous laisse libre arbitre/ (Céline) ». Cet ajout recèle plusieurs stratégies de narration. Par «je vais vous raconter», Sophie, la narratrice, s'adresse au lecteur interpellé par « vous » pour lui signifier qu'elle n'a pas encore commencé l'histoire. Comme ce qu'elle introduit va se rapporter à une histoire surnaturelle (l'intervention d'une mystérieuse voix), elle s'attend à ce que le lecteur se montre sceptique par rapport à son histoire. Elle anticipe alors la réaction de ce dernier en l'invitant à la croire, mais sans jamais l'y forcer.

Pour le remplacement, nous avons identifié des remplacements qui reformulent (RR) et des remplacements formels (RF). Alors que les RF «portent sur les modifications d'ordre graphique et grammatical » (Niwese, 2010 : 406), les RR consistent à « exprimer la même chose en utilisant les termes équivalents sur le plan sémantique » (ibid. : 412) et ce dans une quête apparente des termes plus justes (exemple: " Fon père revenait le week end car il était encore dans l'armée/Ton père, à l'époque fesait le service militaire et il avait la permission le week-end ${ }^{5}$ [Céline] ») ou dans le repositionnement du scripteur dans la prise en charge de son discours (« Et puis, /A sa demande/j’ai quitté mon boulot 
[Céline] »). Du point de vue quantitatif, les RR (54\%) sont plus représentés que les RF (46\%), sauf pour les scripteurs dont la première langue de socialisation n'est pas le français, ce qui montre que les remplacements ne se résument pas à de simples modifications de surface. L'analyse des RF montre que les "participants sont certes animés par une volonté manifeste d'orthographier correctement, mais qu'ils sont confrontés à leurs propres limites" (ibid.: 424). En effet, sur $100 \mathrm{RF}$, on recense 47 suppressions d'erreurs ("le premier jour de ("On m'a / ma/toujours sélectionné»), 23 maintiens d'erreurs («j'etudie à l'etnat / eternant») et 4 maintiens de formes correctes ( $j$ 'ai travaillé comme bucheron / bûcheron/ 15 ans »).

\section{De la première version réécrite à la version publiée}

La comparaison entre la V2 et la V4 permet de poser l'atelier d'écriture comme un observatoire de l'existant, ce qui constitue un enjeu important quand on est en face d'un public hétérogène, dont on ne peut déterminer a priori ce dont il est capable. Dans le cadre du corpus analysé, cette comparaison a révélé que les scripteurs observés maitrisaient plus la macrostructure et la superstructure (Adam, 1992) du récit que des niveaux inférieurs relevant des micro-compétences de la langue écrite (Niwese, 2010 ; 2012).

Dans ce sens, sur le plan morphographique, on relève par exemple des confusions au niveau des morphèmes homophones ou proches phonétiquement (à/a, est/es/et/ai, on/ ont, où/ou, ces/ses/ce/se, son/sont/, etc.) et des difficultés à segmenter des unités du discours («Jaime sa profession » [J'aime sa profession]; Ces pour ça quelle à du fuir la maison [C'est pour ça qu'elle a dû fuir la maison] ; mon chef ma doré beaucoup [mon chef m'adorait beaucoup]; il la eu tout ce que Il voulait [il a eu tout ce qu'il voulait], etc. ).

Si ces défaillances peuvent être reliées au fait que les participants sont, dans leur quotidien, exposés plus à l'oral qu'à l'écrit, d'autres peuvent être dues à la spécificité du français par rapport aux langues premières de certains participants. Ainsi, l'absence, dans certaines langues, du phonème $/ Y /$ ou du phénomène de nasalisation peut justifier la confusion entre $/ \mathrm{Y} /$ et /i/ ou des situations de (dé)nasalisation : « Ma mère repondu Bonjour [Ma mère répondit «bonjour »] »; "A rentra dans la classe j'ai vie pas mal des autres enfants qui avaient de l'armes aux yeux comme moi [En rentrant dans la classe, j'ai vu pas mal d'autres enfants qui avaient des larmes aux yeux comme moi]»; " Or on fond de moi je m'airais bien avoir, un mari [Or, au fond de moi, j'aimerais avoir un mari] », etc.

48 Dans l'ensemble, les difficultés d'ordre morphographique se rapportent plus à des morphèmes grammaticaux (libres et liés) qu'à des morphèmes lexicaux. Ce constat reste valable lorsqu'on considère les aspects ayant trait à la dimension textuelle.

Ainsi, au niveau de la cohésion textuelle, on dénombre moins de difficultés se rapportant à la coréférence et à la coextension (Adam, 2008), qui impliquent des unités lexicales. Par contre, des marqueurs de référence relevant de la grammaire (des reprises pronominales, des marqueurs de genre ou de nombre) «sont sérieusement entravés par les difficultés morphographiques» (Niwese, 2010: 474). Dans ce sens, lorsque, reprenant les propos du personnage de son récit, Côme écrit «je mi suis appliqu ée dans ce sport que j'ai vraiment appréciée ces valeur moral et surtout humaine ", l'accord du participé passé appliquée peut laisser penser, alors que ce n'était pas le cas, que le 
narrateur était une femme. De même, l'usage du déterminant démonstratif à la place $\mathrm{du}$ possessif ne permet pas de voir que les valeurs dont il est question sont celles $d u$ sport.

Du point de vue textuel, les textes produits connaissent les structures d'un récit. Ces textes ont un début (une situation initiale), un milieu (avec transformations et péripéties) et une fin (un dénouement). Ces textes recèlent par ailleurs des séquences textuelles diversifiées.

51 Tenant compte du corpus analysé, nous avons été amenés à conclure «qu'entre le savoir-faire textuel et le savoir-graphier - tous deux constitutifs de la compétence scripturale -, c'est sur cette dernière composante que portent les grandes difficultés » (Niwese, $2010:$ 475).

\section{Vers la construction d'un nouveau dispositif}

Dans cette dernière partie, nous présentons le début du processus de transposition du dispositif expérimenté en milieu scolaire. Précisément, après le rappel du statut de l'écriture de soi à l'école, nous rendrons compte des résultats obtenus après une première analyse de la phase exploratoire.

\subsection{Une écriture de soi délaissée}

L'écriture de soi ne va pas de soi à l'école où l'usage du " je » revêt un statut à la fois ambigu et mouvant (Bishop, 2010). Ambigu parce que la présence de la première personne n'indique pas une «intention d'écrire à partir de son vécu »: le scripteur ne signe pas le pacte autobiographique de son plein gré et l'écriture à la première personne signale bien davantage un genre scolaire (Bishop, 2006). Mouvant parce que, de l'institutionnalisation de la rédaction sous la III ${ }^{e}$ épublique aux sujets se rapportant à la vie personnelle de l'enfant (scolaire ou familiale), le genre a largement évolué, notamment à partir de Freinet avec l'émergence du texte libre et de l'écrit dit authentique.

Mais les écrits libres, comme les écrits de soi, restent marginaux dans les pratiques d'écriture scolaire. Ainsi, par exemple, dans les programmes de 1992, l'écriture autobiographique est envisagée "à travers le portrait, comme un genre littéraire " (ibid.) et non plus comme une expression personnelle. Si la première personne n'a pas disparu, dans les programmes de 2002, «le vécu est plus un réservoir de connaissances que l'occasion d'une expression personnelle » (Bishop, 2010) et si les maitres utilisent régulièrement les souvenirs personnels comme matériau exploitable pour la production d'écrit, ils sont souvent gênés de solliciter ce matériau intime et d'y intervenir (ibid.).

Autant l'écriture de soi régresse en contexte scolaire, autant le « rapprochement des écrits scolaires avec la littérature » (Bishop, 2006) s'accélère et se trouve encouragé par les textes officiels de 2002. Ceux-ci proposent une approche littéraire des œuvres pour la jeunesse, qui favorise la production des écrits d'imitation de textes classiques et/ou patrimoniaux. Cet apprentissage de l'écriture par l'imprégnation des classiques se déroule dans un contexte où le modèle traditionnel de la rédaction reste dominant, ce qui a pour conséquence que l'écriture continue d'être considérée comme ne faisant pas 
l'objet d'un "véritable enseignement » et-comme la synthèse "magique » des autres enseignements du français (Reuter, $2002: 51-52$ ).

On remarque donc que les avancées de la recherche en didactique de l'écriture n'ont pas encore modifié les habitudes. Ainsi, par exemple, même si les apports de la génétique textuelle et l'avènement des brouillons en tant qu'objets didactiques ont permis aux enseignants de réfléchir sur les bénéfices de la réécriture, rares sont les activités d'écriture qui favorisent réellement cette pratique. De même, si nombre de travaux récents ont mis l'accent sur l'importance de l'articulation entre la lecture et l'écriture dans l'apprentissage de l'écriture, cette articulation reste peu effective dans les classes.

Si l'école semble délaisser les principes de la réécriture génétique, elle s'intéresse paradoxalement à la récriture intertextuelle en tant que contact authentique et privilégié avec l'œuvre littéraire : or, si la démarche n'est pas la même, la seconde, en tant que transformation d'un hypotexte, appelle des réécritures successives, "une somme de préparations, de corrections et de ratures, de variantes successives" (Gignoux, 2006).

\subsection{Contexte d'exploration}

La phase d'exploration s'est déroulée entre janvier et février 2013, dans deux établissements situés en zone périurbaine bordelaise du même secteur de la carte scolaire. Deux classes - une de cours moyen (CM1-CM2) et une autre de sixième - ont fait l'objet de notre observation. Ces classes ont été choisies parce qu'elles présentent des points de convergence avec les groupes d'adultes, dont il a été question dans la première étude.

59 En effet, dans ces classes, le public était hétérogène par ses origines sociales, les écoles de provenance (pour le collège), les pratiques antérieures d'écriture et de lecture, et les résultats scolaires. Certains élèves éprouvaient des difficultés d'ordre personnel comme des problèmes de concentration. En outre, les enseignants de ces classes mettaient en œuvre des projets d'écriture visant la production de textes narratifs à partir de la réécriture de contes et prévoyant la socialisation des textes produits. Au cours de cette phase, notre rôle a été d'observer et d'enregistrer sans intervenir des séances d'écriture, en lien avec les objets du programme, entièrement conçues et mises en œuvre par les enseignants.

\subsection{Dispositifs observés}

60 Les projets d'écriture mis en œuvre visaient la production des contes détournés à partir d'un hypotexte commun pour le collège et de plusieurs hypotextes pour le primaire. En petits groupes de trois à quatre élèves, les élèves de sixième devaient réécrire, de façon collaborative, Le Petit Poucet, ceux de CM un des contes auxquels il est fait allusion dans l'album Le Tunnel d'Anthony Browne. Le détournement pouvait porter sur la transposition spatio-temporelle, sur la dégradation et/ou sur l'inversion (ConnanPintado, 2010). Dans les deux classes, la production du conte a été précédée par la réactivation des contes hypotextuels et par un travail sur la superstructure du conte. La classe de sixième est allée plus loin en travaillant, avant la réécriture, sur 
l'identification des procédés de détournement, à partir de situations initiales de contes extraits de la littérature de jeunesse.

Mis à part les phases de réactivation, les textes ont été produits en sept séances d'une heure pour le collège et en cinq séances de 40 minutes pour le $\mathrm{CM}$. La socialisation des productions initialement prévue devait être organisée en plusieurs temps : la lecture des textes au groupe de scripteurs et à la classe dans le temps de la révision collective ainsi que, pour la classe de sixième, la publication des textes au centre de documentation et d'information (CDI) du collège. En réalité, si la lecture des textes s'est faite régulièrement au sein de chaque groupe de scripteurs, seule la classe de $\mathrm{CM}$ a conservé la lecture des contes au groupe-classe et l'idée de publication a été abandonnée.

\subsection{Quelques résultats intermédiaires}

62 Notre analyse a porté sur un corpus composé de 11 textes ( 7 de sixième et 4 de $\mathrm{CM}$ ), chaque texte étant recueilli en deux versions, une version initiale relue et annotée par l'enseignant et une version définitive. Sont aussi analysés 11 entretiens d'explicitation avec chaque groupe-auteur, un entretien d'explicitation avec les deux enseignants et une soixantaine d'heures d'enregistrement audio de séances d'écriture.

Au niveau du rapport à l'écriture, c'est la dimension affective qui semble avoir été le plus touchée. La quasi-totalité des élèves interrogés dit avoir apprécié l'expérience d'écriture collaborative en déclarant qu'ils ont passé plus de temps à l'écriture d'un texte qu'ils ne le feraient tout seuls ou qu'ils ont produit des textes plus longs que d'habitude : «On passe plus de temps [que] sur la rédaction »; " nos rédactions sont moins longues "; " on a fait deux pages entières, cinq pages même ».

On trouve aussi, dans les déclarations des élèves, des éléments relevant de leur conception de l'écriture. Selon eux, s'ils ont réussi à produire leurs textes, c'est grâce à l'écriture en groupe. Quand on écrit à plusieurs mains, les scripteurs mutualisent leurs compétences et le texte rédigé résulte d'une négociation :

"Je pourrais mettre plein d'idées, mais ça aurait pas vraiment sens ; ça aurait pas vraiment du sens parce que quand on est tous ensemble, on est plusieurs cerveaux à réfléchir, et donc si quelqu'un donne une idée qui est fausse qu'on voudrait la mettre, les autres sont là pour dire : "non attention, faudrait modifier comme ça" et donc on modifie et après on pourrait placer l'élément » (Olivier, sixième, entretien d'explicitation).

Comme elle requiert une négociation entre pairs et une justification de points de vue défendus, l'écriture collaborative favorise une posture non seulement métatextuelle, mais aussi métagénérique. Par exemple, dans l'extrait qui suit, la négociation porte sur le maintien ou non de l'ogre dans le conte modernisé. Dans ce conte, la fugue du petit Poucet cancre conduit celui-ci sur un terrain de basket sur lequel les joueurs sont équipés de prothèses :

Antoine : $Y$ en a un avec des grandes bottes.

Pierre : Ah oui, les bottes de sept lieux.

Antoine : Oui mais c'est des bottes, elles ont été montées.

Mathilde : Ah oui en prothèses.

Pierre : Oui, en réalité, c'était des prothèses.

Antoine : Oui, un peu comme des échasses.

Marie : Oui, mais si on met les bottes de sept lieux, on met l'ogre.

Pierre : Ben.. 
Marie : Ben parce que ça va dans l'histoire de l'ogre.

Pierre : Peut-être qu'il les trouve dans...

Maire : C'est comme si on supprime l'argent, eh ben on supprime l'ogre.

Paul : dans le magasin souvenir.

Marie : si on met l'argent et qu'on met pas l'ogre, ça va pas ; tu peux / tu vas pas,

enfin ça va pas déjà dans la vraie histoire.

Pierre : Dans la vraie histoire, il est pauvre le petit Poucet et il y a un ogre.

Marie : Déjà non il est riche le petit Poucet.

Pierre : Donc, il n'y a pas d'ogre. expérimenté auprès d'un public adulte en difficulté, atelier dont les lignes de force sont le texte narratif, la socialisation de celui-ci et la réécriture. L'analyse des déclarations et des productions des participants a montré que ce dispositif a permis à ces derniers d'améliorer leur rapport à eux-mêmes, aux autres et à l'écriture, et d'identifier les limites et les compétences des scripteurs en matière d'écriture.

Encouragés par ces résultats, nous avons entamé la transposition du dispositif expérimenté en milieu scolaire. L'analyse de la phase d'exploration a permis de constater que, moyennant quelques adaptations, ce dispositif peut être transposé à l'école. 
71 Ainsi, pour des raisons développées précédemment, le dispositif transposé sera centré sur la production de textes narratifs en lien avec la construction du genre, ce qui permettra, entre autres, d'initier les élèves à la production de plusieurs séquences textuelles. Le nouveau dispositif devra aussi intégrer plus nettement le vécu des élèves, mais aussi leurs savoirs théorico-réflexifs et leur imaginaire (Reuter, 2002) en générant notamment des situations d'intertextualité élargie qui inclurait, dans le processus de production, des références artistiques et culturelles différentes - littérature patrimoniale ou de jeunesse, jeux, films, pièces de théâtre - à la fois comme objets sémiotiques et comme sources de création (Bazile, à paraitre).

Tel le dispositif expérimenté, le dispositif transposé sera envisagé dans une logique de la pédagogie du projet (Huber, 2005) et visera une large socialisation des textes produits, socialisation qui permettra aux élèves de s'engager réellement dans la réécriture et de ne pas considérer cette pratique comme une simple mise au propre du brouillon. Faire réécrire passera également par un travail sur la place et les enjeux de la réécriture dans le processus d'écriture.

Enfin, le nouveau dispositif alternera des moments d'écriture collaborative et individuelle, permettant ainsi de tirer profit des bénéfices de l'écriture collaborative tout en palliant son incapacité à mesurer des progrès individuels. Dans ce sens, les élèves pourront construire ensemble une superstructure du texte narratif à produire (conte, nouvelle, fait divers, etc.) ou travailler sur des stéréotypes du genre concerné et/ou sur leurs avatars avant que chacun ne procède à l'écriture de sa propre histoire.

\section{BIBLIOGRAPHIE}

ADAM, J.-M. (1992) : Les textes : types et prototypes, Paris, Nathan.

- (2008) : La linguistique textuelle, Paris, A. Colin.

ANDRÉ, A. (1989) : Babel heureuse. L'atelier d'écriture au service de la création littéraire, Paris, Syros (coll. « Alternatives »).

BARRÉ-DE MINIAC, C. (2000) : Le rapport à l'écriture, Villeneuve d'Ascq, Presses universitaires du Septentrion.

BAZILE S. (à paraitre) : « Le tchat (clavardage) comme matériau d'écriture dramatique : réécritures littéraires de l'instantané », actes du colloque Vers une " multilecture » et une " multiécriture » littéraire? Des voies multimodales d'accompagnement de la littérature en classe, $14^{\mathrm{es}}$ rencontres des chercheurs en didactique des littératures, mai 2012.

Bisнop, M.-F. (2006) : « Les écritures de soi à l'école primaire : bref historique d'un genre scolaire », Repères, 34, p. 21-40.

- (2010) : «Racontez vos vacances... ». Histoire des écritures de soi à l'école primaire (1882-2002), Grenoble, Presses universitaires de Grenoble. 
BUCHETON, D. (1992) : Écriture-Réécritures. Récits d'adolescents, thèse de doctorat, Paris, Université Paris-Descartes.

- (1995) : Écriture, réécriture, récits d'adolescents, Berne, P. Lang.

CHARTRAND, S.-G. \& BLASER, C. (dirs) (2008) : Le rapport à l'écrit. Un outil pour enseigner de l'école à l'université, Namur, Presses universitaires de Namur.

CONNAN-PINTADO, C. (2010) : Lire des contes détournés à l'école, Paris, Hatier.

DABÈNE, M. (1991). « Un modèle didactique de la compétence scripturale », Repères, 4, p. 9-22.

FABRE-COLS, C. (2002) : Réécrire à l'école et au collège. De l'analyse des brouillons à l'écriture accompagnée, France, ESF.

FENOGLIO, I. \& BOUCHERON-PÉTILLON, S. (2002) : « Avant-propos », Langages, 147, p. 3-7.

GANASCIA, J.-G., FENOGLIO, I. \& LEBRAVE, J.-L. (2004) : « Manuscrits, genèse et documents numérisés. EDITE : une étude informatisée du travail de l'écrivain », Temps et documents, 8 (4), p. 91-110.

GIGNOUX A.-C., (2006) : «De l'intertextualité à l'écriture », Cahiers de Narratologie, 13 (en ligne : http:// narratologie.revues.org/329).

GRÉSILLON, A. (1994) : Éléments de critique génétique. Lire les manuscrits modernes, Paris, Presses universitaires de France.

GUEUNIER, N. (1993) : Le français du Liban. Cent portraits linguistiques, Aix-en-Provence, Institut d'études créoles et francophones.

HUBER, M. (2005) : Apprendre en projets. La pédagogie du projet-élèves, Lyon, Chronique sociale.

LAFONT, J. (1999) : Pour une ethnolinguistique des ateliers d'écriture. Analyse de pratiques sur plusieurs terrains, thèse de doctorat, Tours, université François-Rabelais.

LAFONT-TERRANOVA, J. (2009) : Se construire, à l'école, comme sujet-écrivant. L'apport des ateliers d'écriture, Namur, PUN.

LEJEUNE, P. (1996) : Le Pacte autobiographique, Paris, Éd. Le Seuil.

NIWESE, M. (2010) : L'atelier d'écriture : un dispositif didactique pour apprendre à écrire à un groupe multiculturel d'adultes en reprise de formation, thèse de doctorat, Louvain-la-Neuve, Université catholique de Louvain.

- (2011). « Quel dispositif pour apprendre à écrire à un public multiculturel en difficulté ? », La Lettre de l'AIRDF, 49, p. 36-39.

- (2012) : «Balises pour un atelier d'écriture adapté à un public adulte en difficulté », Lidil, 45, p. 123-137.

REUTER, Y. (2002) : Enseigner et apprendre à écrire, Paris, ESF.

RICCEUR, P. (1985) : Temps et récit 3. Le temps raconté, Paris, Éd. Le Seuil.

STERCQ, C. (2009) : « Des bonnes raisons de refuser l'alphabétisation », Le Journal de l'Alpha, 167-168, p. $100-102$.

VERHOEVEN, M. (2002) : École et diversité culturelle, Bruxelles/Louvain-la-Neuve, Bruylant/Academia (coll. « Sybidi »). 


\section{NOTES}

1. Ce décret a été publié au JO du 28 juillet 2013.

2. L'énonciateur «nous » pour la partie concernant la recherche en milieu adulte renvoie à l'un des auteurs de cet article.

3. Voir Y. Reuter (2002: 137-145).

4. Les segments textuels ajoutés sont repris entre deux barres obliques. Ceux qui intéressent directement notre analyse sont mis en gras.

5. Le terme remplacé est biffé, tandis que celui qui le remplace est repris entre deux barres obliques.

\section{RÉSUMÉS}

L'école accueille de plus en plus des populations hétérogènes, dont la maitrise de la langue écrite est défaillante, alors que celle-ci participe à la construction des savoirs dans toutes les disciplines. Cette hétérogénéité, qui est notamment due à l'évolution des publics, ne permet pas de déterminer a priori les compétences des élèves, alors que l'identification de celles-ci est un préalable pour la mise en œuvre d'interventions didactiques efficaces. La recherche présentée ici fait partie d'un projet global qui cherche à pallier cette situation en élaborant, via un dispositif d'atelier d'écriture, une méthodologie d'identification et d'analyse des difficultés et des compétences en matière d'écriture auprès des élèves du cycle 3 et de sixième au collège, niveau qui semble révéler des situations de grande hétérogénéité. Cet article porte principalement sur un dispositif d'atelier d'écriture conçu et expérimenté auprès d'un public adulte en difficulté, dispositif en cours de transposition à l'école. Outre la présentation de ce dispositif, il s'agira de montrer comment celui-ci permet d'identifier et de travailler la compétence scripturale dans toutes ses composantes. Il sera enfin question de quelques résultats obtenus après la première phase du processus de transposition, résultats qui permettront de tracer les grandes lignes du nouveau dispositif.

School welcomes more and more heterogeneous populations. Their mastering of writing language is failing while it contributes to the building of knowledge in every branch of learning. This heterogeneity, which is notably due to the sociological evolution of the school population, prevents teachers from determining students' skills a priori when their identification is a prerequisite for implementing efficient pedagogic actions. Our research is part of a global project aiming to compensate this situation by developing, through a writing workshop, a methodology to identify and analyze writing problems and skills of pupils aged 9 to 12, these pupils being in grades which seem to reveal conditions of great heterogeneity.

This article focuses on a writing workshop both devised and experimented with adults experiencing writing as well as identity difficulties, a workshop being transposed to school younger learners. In addition to the presentation of this project, we will show how it enables to identify and work on all the components of the scriptural competence. Then, we will present some results from the first stages of this transposition process, results which will outline the main features of the future pedagogic device for school. 
INDEX

Keywords : writing workshop, scriptural competence, heterogeneous school population, rewriting, narrative text

Mots-clés : atelier d'écriture, compétence scripturale, public hétérogène, réécriture, texte narratif

\section{AUTEURS}

\section{MAURICE NIWESE}

LACES (EA 4140), ESPÉ, université de Bordeaux

\section{SANDRINE BAZILE}

CLARE (EA 4593), ESPÉ, université de Bordeaux 